inspirierten Deutungen, denen das »Dritte Reich« wie ein über Deutschland hereingebrochenes Fremdregime mit einer im Grunde geringen Zahl von »Kollaborateuren « und einem Heer harmloser Mitläufer erschienen war, trat seitdem ein wachsendes Bewußtsein für die Dimensionen des Verbrechens der "Endlösung", trat kritische Aufklärung über die gesellschaftliche Verankerung des Nationalsozialismus, seine Trägerschichten und die Verstrickung der auch nach 1945 wieder präsenten Funktionseliten.

Vorausgegangen war dieser Entwicklung hin zu einer ernsthaften überindividuellen Auseinandersetzung mit dem Nationalsozialismus jedoch - und dies gilt es festzuhalten - eine Phase größter Milde für die Individuen, die gewissermaßen erst die Basis für einen offeneren Umgang mit der Vergangenheit schuf: die Phase der Amnestie- oder Vergangenheitspolitik, deren politische Fehler und moralische Versäumnisse das geistige Klima in der Bundesrepublik nachhaltig prägten.

\title{
Ulrike Homann \\ Die Rechtsbeugungsprozesse gegen ehemalige DDR-Richter und Staatsanwälte vor dem Bundesgerichtshof
}

In der DDR wurden rund 200000 Menschen $^{1}$ aus politischen Gründen verurteilt und eingesperrt, meist nach Verfahren zur "Zusammenhaltung des Staatsvolkes « und insbesondere wegen Republikflucht. ${ }^{2}$ Gegen die dafür verantwortlichen Richter und Staatsanwälte wurden nach 1989 über 12000 Verfahren eingeleitet. ${ }^{3}$ Inzwischen liegen mehrere Urteile des BGH zu diesem Komplex vor. ${ }^{4}$ Grundlegend sind vor allem zwei: Im ersten Urteil vom I 3. I 2. I 993 entwickelte der BGH seine grundsätzlichen Positionen zur Frage der Rechtsbeugung durch Anwendung von DDR-Recht. In seiner Entscheidung vom I 5.9. I995 führt der BGH die Maßstäbe im Detail aus, nach denen Rechtsbeugung in der DDR im Einzelfall ermittelt werden soll. Dabei hat der Gerichtshof auch auf die Kritik an seinen früheren Entscheidungen reagiert.

I Friedrich Christian Schroeder, Geschichtsbewaltigung durch Strafrecht?, in: DRiZ i 996, S. 8 I.

2 Christoph Schaefgen, „DDR-Regierungskriminalitat» - Erscheinungsformen und Probleme, in: Recht und Politik 1992, S. 194.

3 BT-Drucks, $12 / 8402$, S. 4

4 Insbesondere: Urteil v. 13. 12. 1993 (5 StR 76/93), in: BGHSt 40, 30 (NJW 1994, S. 529-532); Urteil v. 9. 5. 1994 (5 StR 354/93), in: NJW 1994, S. 3238-3242; Beschluß v. 10.8.1994 (3 StR 252/94), in: NJW 1994, S. 456; Urteil v. 6. 10. 1994 ( 4 StR 23/94), in: NJW 1995, S. 64-67; Urteil v. 5.7.95 (3 StR 605/94), in NJW 1995, S. 2734-2738; Urteil v. 15.9. 1995 (s StR 713/94), in: NJW 1995, S.3324-3332; Urtell v. 15.9. 1995 (5 StR 168/95), in: NJ 1996, S. 153 ; Urteil v. 15.9. 1995 (5 StR 23/95), in: NJ 1996, S. 152 (beide in der NJ stark gekurzt); Urteile v. 1 5.9. 1995 (5 StR 642/94) und (s StR 68/95), beide unveroffentlicht; Urteile v. I 5. I I. 1995 (3 StR 68/95) und (3 StR 527/94), beide unveroffentlicht; Urteil v. I5. I I. 1995 (3 StR 527/94), in: DtZ 1996, S. 92-96; Urteil v. 16. 11. 1995 (s StR 747/94), in: NJW 1996, S. 857-865; Urteil v. 30. I1. 1995 ( 4 StR 777/94), in: NStZ-RR 1996, S. 65-69; Urtell v. 30.11. 1995 (4 StR 714/94), in: NStZ-RR 1996, S. 69-7I. 
a. Die Strafbarkeit vor bundesdeutschen Gerichten

Nach Ansicht des BGH können Richter und Staatsanwälte der ehemaligen DDR wegen Rechtsbeugung verfolgt werden. Ferner gilt die Verfolgbarkeit auch für Delikte, die in Tateinheit mit der Rechtsbeugung begangen wurden.' Der Einwand der Angeklagten, "daß Handlungen, die Angehörige von Staatsorganen der DDR in Ausübung ihres Dienstes vorgenommen haben, der Aburteilung durch Gerichte der Bundesrepublik Deutschland entzogen seien ${ }^{6}$, wird zurückgewiesen.

Zunächst wird festgestellt, daß Rechtsbeugung, im bundesrepublikanischen Strafrecht mit $\$ 336$ StGB unter Strafe gestellt, auch in der DDR nach $₫ 244$ DDR-StGB strafbar war. Dieser Umstand werde nicht dadurch beeinflußt, daß es sich um zwei unterschiedliche Rechtsordnungen handelte ${ }^{7}$ : Art. $3 \times 5$ Abs. 2 EGStGB i. V. mit $\mathbb{} 2$ StGB regelte die Anwendung des $\$ 336$ StGB auf die sogenannten "Alttaten" der DDR. So sei nach $\$ 2$ Abs. I StGB das Recht maßgeblich, welches zur Tatzeit gegolten habe, also $\$ 244$ DDR-StGB. $\$ 336$ StGB komme nur zum Tragen, wenn er nach $\$ 2$ Abs. 3 StGB das mildere Gesetz darstelle. ${ }^{8}$ Ebenso will der BGH auch die Vorsatzfrage behandelt sehen. Da die bundesrepublikanische Rechtsprechung inzwischen von der Strafbarkeit des indirekten Vorsatzes ausgeht ${ }^{9}$, während das DDRGesetz nur von einer wissentlichen Rechtsbeugung spricht, soll auch hier $\$ 244$ DDR-StGB das mildere Gesetz sein. ${ }^{10}$ Praktisch bedeutet das, daß sich die Richter zunächst nach $\$ 244$ DDR-StGB strafbar gemacht haben müssen und dann nachgewiesen werden muß, daß diese Straftat auch nach $\$ 336$ StGB strafbar ist. Bei Nichterfüllung des Tatbestandes von $\$ 336$ StGB kommt also auch $\$ 244$ DDR-StGB nicht zur Anwendung. ${ }^{\text {.1 }}$

Auch Staatsanwälte können sich nach Ansicht des BGH der Rechtsbeugung schuldig machen. Im $\$ 244$ DDR-StGB werden sie explizit aufgeführt, während es in der Bundesrepublik durchaus eine Auseinandersetzung über diesen Streitpunkt gab. Die Grundvoraussetzung der Strafbarkeit bestehe darin, daß der Staatsanwalt im Untersuchungsverfahren durch die Möglichkeit der Einstellung des Verfahrens oder der Anklageerhebung mit der Entscheidung einer Rechtssache betraut sei und einen gewissen Grad sachlicher Unabhängigkeiten genieße. ${ }^{12}$ Da weder Verjährung noch Amnestien im Wege stehen, hält der BGH es also grundsätzlich für möglich, Richter und Staatsanwälte der ehemaligen DDR wegen Rechtsbeugung vor bundesdeutschen Gerichten anzuklagen.

In seinem ersten Urteil charakterisierte der BGH das DDR-Rechtssystem, um auf Unterschiede der Stellung von Richtern in der alten Bundesrepublik und der DDR hinzuweisen. ${ }^{13} \$ 336 \mathrm{StGB}$ bezieht sich auf den unabhängigen, nur dem Gesetz un-

5 BGH, NJW r995, S.65.

6 BGH, NJW 1994, S. 3239

7 Vgl. BGH, Urteil v. 11.6. I991 (s StR I80/91), NJW 1991, S. 2300, bei dem die Strafbarkeit einer Eınfuhrung von Drogen in die DDR auch nach der Wiedervereinigung bestehen bleibt.

8 BGH, NJW 1994, S. 3239 .

9 Seit der Strafrechtsreform von 1975 geht auch der BGH von der Geltung des dolus eventualis aus. Die vorangegangene Praxis, nur den direkten Vorsatz zu bestrafen, war in dieser Konkretisierung vom Gesetzestext nicht gedeckt und hatte zu der Grundsatzdiskussion gefuhrt.

Io $\mathrm{BGH}, \mathrm{NJW}$ i $995, \mathrm{~S} .65$.

II BGH, NJW 1994, S. 529-530.

12 BGH, NJW 1994, S. 3240; BGH, NJW 1995, S. 3325 ; BGH, NJ 1996, S. 152 -153 sowie s StR 68/94. Gegen die Staatsanwalte als Tater i.S. des $\ 336 \mathrm{StGB} z$. B. Olaf Hohmann, Zur Rechtsbeugung durch DDR-Staatsanwalte, in: NJ 1995, S. $128-132$.

I3 Empfehlenswert zur Charakterisierung des DDR-Rechtssystems: Hubert Rottleuthner (Hg.), Steuerung der Justiz in der DDR. Einflußnahme der Polıtık auf Richter, Staatsanwalte und Rechtsanwailte, Koln 1994. 
terworfenen Richter. Zwar erklärten ein Lehrbuch und ein Kommentar der ehemaligen DDR zum Strafrecht, daß $\mathbb{2} 24$ DDR-StGB »der Gewährleistung des Grundsatzes der Gleichheit vor dem Gesetz [...] sowie der Sicherung einer in allen Fragen gerechten und gesetzlichen Rechtsprechung ${ }^{14}{ }^{14}$ diene und "den hohen Rang und die prinzipielle Bedeutung zum Ausdruck [bringe, U.H.], die in der sozialistischen Gesellschaft der Wahrung der Gesetzlichkeit und der Würde des Menschen

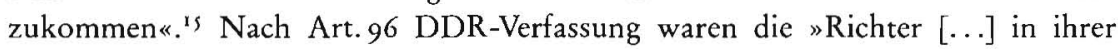
Rechtsprechung unabhängig.«Sie sollten »nur an die Verfassung, die Gesetze und andere Rechtsvorschriften der Deutschen Demokratischen Republik gebunden $\aleph^{16}$ sein. Der BGH führte aber aus, daß die Gerichte als Organe mittels Rechtsprechung die staatliche Macht der Arbeiterklasse ausübten und fest in das einheitliche System der Machtausübung eingebunden waren. Wenn von den Rechten der Bürger die Rede gewesen sei, dürfe man nicht vergessen, daß der "Ausgangspunkt jeder auf den einzelnen Bürger bezogenen Staatstätigkeit, auch der Rechtsprechung, die Annahme [war, U.H.], daß alles, was der Entwicklung und Festigung der sozialistischen Gesellschaft diene, zugleich den Interessen des einzelnen « entspreche. ${ }^{77}$ Der Zusatz "sozialistisch «, führte der BGH aus, orientierte die Gesetzesanwendung auf das Staatsziel, die Verwirklichung eines sozialistischen Staates. Da die SED Verfassungsrang besaß, weil sie die Inhalte des Sozialismus und damit die sozialistische Komponente der Gesetzgebung definierte, war sie nach der Ansicht des BGH der Rechtsprechung übergeordnet. Darin sah der BGH eine Aufhebung der in der Verfassung vorgeschriebenen Unabhängigkeit der Richter und eine starke äußere Beeinflussung. Ferner wies der BGH auf einen weiteren charakteristischen Punkt hin: "Einheitlichkeit der Rechtsprechung hatte einen weit höheren Stellenwert als in der Bundesrepublik. $\ll^{18}$

Die Feststellung dieser tiefgreifenden Unterschiede veranlaßte den BGH aber nicht, von einer grundsätzlichen Strafbarkeit abzusehen, da »die Rechtsprechung unabhängig von diesem politischen Bezug auch dazu diente, ein geordnetes Zusammenleben der Menschen zu regeln. «'9 Der BGH stützte sein Ergebnis darauf, daß laut Einigungsvertrag die in der DDR gefällten Urteile in der Regel ihre Gültigkeit behalten sollten. Außerdem sei aus der Möglichkeit, daß Richter der ehemaligen DDR in ein Richteramt nach geltendem Recht berufen werden konnten, abzuleiten, daß von den Verhandlungsparteien eine (eingeschränkte) Vergleichbarkeit angenommen worden sei. $^{20}$

14 StGB der DDR, Kommentar, hrsg. v. Ministerium der Justiz, Berlin 5. Aufl. 1987, S. 516.

is Strafrecht. Besonderer Tell. Lehrbuch, hrsg. v. der Sektion Rechtswissenschaft d. Humboldt-Universitat zu Berlin und der Akademie fur Staats- und Rechtswissenschaft der DDR Potsdam-Babelsberg, Berlin I981, S. 232.

I6 Siegfried Mampel, Die sozialistische Verfassung der DDR. Text und Kommentar, Frankfurt a. M. 1982, S. 1273 .

17 BGH, NJW 1994, S. 530 .

18 Ebd.

19 Ebd., S. 531. Eben solches gilt auch fur Staatsanwàlte, so z. B. BGH, NJW 1994, S. 3239.

20 Eine Vergleichbarkett wird abgelehnt von Thomas Vormbaum, Zur strafrechtlichen Verantwortlichkeit von DDR-Richtern wegen Rechtsbeugung, in: NJ 1993, S. 212-215. 
Um einen Verstoß gegen das Rückwirkungsverbot des Art. I03 Abs. 2 GG zu verhindern, grenzt der BGH den Straftatbestand der Rechtsbeugung stark ein. Wie schon in seiner früheren Rechtsprechung ${ }^{21}$ weist der BGH in seinen Entscheidungen zu DDR-Rechtsbeugungen daraufhin, "daß $\$ 336$ StGB nicht schlechthin jede unrichtige Rechtsanwendung, sondern nur die Beugung des Rechts treffe ${ }^{22}$. Im Urteil vom 15.9.1995 formulierte er diesen Grundsatz noch schärfer: "Nur der Rechtsbruch als elementarer Verstoß gegen die Rechtspflege soll unter Strafe gestellt werden. ${ }^{23}$ Der BGH sah sich zu einer solchen Festlegung genötigt, um Eingrenzungsproblemen beim subjektiven Tatbestand vorzubeugen. Er befürchtete, über den Rechtsbeugungstatbestand könnten vermehrt Entscheidungen angegriffen werden, die auf dem normalen Rechtsweg nicht zur Zufriedenheit der einen oder anderen Partei geführt hatten. Die »Sperrwirkung « des Rechtsbeugungsparagraphen in bezug auf andere Straftatbestände soll auch für DDR-Richter gelten. ${ }^{24}$

Als Quintessenz seiner Ausführungen möchte der BGH nur in Fällen bestrafen, »in denen die Rechtswidrigkeit der Entscheidung so offensichtlich war und insbesondere die Rechte anderer, hauptsächlich ihre Menschenrechte, derart schwerwiegend verletzt worden sind, daß sich die Entscheidung als Willkürakt darstellt « ${ }^{25}$. Willkürakte sind nach Ansicht des BGH unter drei Gesichtspunkten möglich:

- die Überdehnung des Straftatbestandes (»offensichtliches Unrecht»),

- unerträgliches Mißverhältnis zwischen der verhängten Strafe und der abgeurteilten Handlung,

- schwere Menschenrechtsverletzung durch die Art und Weise des Verfahrens (»Ausschaltung des politischen Gegners $«$ ). ${ }^{26}$

Diese Begrenzung der Rechtsbeugung nur auf »schwerwiegendste Menschenrechtsverletzungen ${ }^{27}$ steht in deutlichem Widerspruch zu beiden Gesetzestexten. ${ }^{28}$

Eine weitere Restriktion des BGH besteht darin, daß bei der Verurteilung von DDRRichtern die Auslegungsmethoden der DDR mitberücksichtigt werden sollen. Zu bestrafen sei, wer als Richter oder Staatsanwalt die Straftatbestände unter Überschreitung des Gesetzeswortlauts angewendet oder unter Ausnutzung ihrer Unbestimmtheit überdehnt habe. Sei die Handlung des Richters vom Wortlaut eines Gesetzes gedeckt gewesen, auch in seiner Mehrdeutigkeit, bestehe keine Gesetzwidrigkeit i.S. des $\$ 244$ DDR-StGB. Bei der Berücksichtigung der Eigentümlichkeiten des DDR-Rechtssystems sei zu beachten, daß die Rechtsprechung eng mit politischen Zielsetzungen gekoppelt gewesen sei. Sei der Richter von diesen Einflüssen

${ }_{21}$ Vgl. BGH, Urteil v. 29.7. 1986 (1 StR 330/86), BGHSt 34, 146-1 49 und BGH, Urteil v. 23. 5. 1984 (3 StR 102/84), BGHSt 32, 357-365.

22 BGH, NJW 1994, S. 531 .

23 BGH, NJW 1995, S. 3325.

24 Die Geltung der Sperrwırkung ruft Erinnerungen an die unruhmliche Ahndung von NS-Justizverbrechen wach: "Die Strafbarkeit wegen Totung setzt die gleichzeitige Feststellung einer von ihnen begangenen Rechtsbeugung [...] voraus. " (Gustav Radbruch, Gesetzliches Unrecht und ubergesetzliches Recht, in: ders., Rechtsphilosophie, S. 328.) Mit der Vorgabe, daß die Rechtsbeugung mit direktem Vorsatz begangen worden sein mußte, bestand fur belastete Richter die Moglichkeit, sich auf ihre » Rechtsblindhett« zu berufen, d. h. daß sie ihre Entscheidung zu dem Zeitpunkt, da sie sie fallten, fur Recht gehalten hätten. Die Folgen sind bekannt und inzwischen dokumentiert. S. z. B. Ingo Muller, Furchtbare Juristen. Die unbewaitigte Vergangenheit unserer Justiz, Munchen 1989.

$25 \mathrm{BGH}, \mathrm{NJW}$ r 994, S. 532 .

26 Ebd.; s. a. BGH, NJW 1995 , S. 3326.

27 BGH, NJW 1994, S. 532.

$28 \mathrm{Vgl}$. Stefan Bandel, Anmerkungen zum ersten Rechtsbeugungsurteil, in: NStZ I994, S. 439. Ausfuhrliche Darstellung zu den historischen Wandlungen in der Handhabung des Rechtsbeugungsparagraphen von Manfred Seebode, Rechtsbeugung und Rechtsbruch, in: JR 1994, S. I-6. 
gelenkt worden, sei daraus noch nicht unmittelbar eine Strafbarkeit abzuleiten. Dies sei darauf zurückzuführen, daß der Gesetzesbegriff der DDR, der dem Merkmal der Gesetzwidrigkeit i.S. des $\$ 244$ DDR-StGB zugrunde liege, in Literatur und Rechtsprechung wenig geklärt und durch Theorie und Praxis der »sozialistischen Gesetzlichkeit « nachhaltig verdunkelt worden sei. Bei der Auslegung von Normen komme es auf die Auslegungsmethoden der DDR, nicht auf die der Bundesrepublik Deutschland an. ${ }^{29}$ Dabei seien die sogenannten "Orientierungen « des Obersten Gerichts insoweit zu beachten, als sie mit der - unter Umständen extensiven Auslegung eines gesetzlichen Straftatbestandes noch vereinbar seien. Anders als im nationalsozialistischen Führerstaat habe es in der DDR keine Doktrin gegeben, nach der bloßer Wille von Inhabern staatlicher Macht Recht schaffen könne. ${ }^{30}$

Wenn der BGH eine Auslegung nach den Maßgaben der DDR, u. a. den »Orientierungen" des Obersten Gerichts, für zulässig hält, besteht die große Gefahr, den politischen Impetus der ehemaligen DDR-Machthaber neuerlich zu legitimieren. ${ }^{31}$ Das kann nicht Aufgabe einer rechtsstaatlichen Rechtsprechung sein, besonders da der BGH an anderer Stelle selbst erklärt hat, daß der Richter, der nach $\$ 2 \mathrm{StGB}$ ein Gesetz der DDR anzuwenden habe, sich nicht an überholten politischen Anschauungen orientieren dürfe, "vielmehr sei das Gesetz unter Beachtung geltenden Verfassungsrechts und der Grundsätze rechtsstaatlichen Strafens auszulegen « ${ }^{32}$. Einmal mehr entstammt eine solch deutliche Position nicht dem Urteil gegen einen Juristen..$^{33}$

Die restriktive Handhabung des Rechtsbeugungstatbestands und die Auslegung mit den Methoden der DDR begründet der BGH mit dem Schutz des Vertrauens, den der angeklagte Richter oder Staatsanwalt zu Zeiten der DDR in deren Gesetze haben durfte. Dabei sei zu beachten, daß sich das geschriebene Recht der DDR nicht am Grundgesetz der Bundesrepublik orientiert habe und der Rechtsstaat mit seiner Wertordnung den DDR-Juristen fremd gewesen sei. ${ }^{34}$ Bemmann merkt hierzu treffend an, daß die Irrtumsregelung den Vertrauensschutz «zur Genüge «35 leiste.

29 BGH, NJW I994, S. $531-532$.

30 BGH, NJW I 995, S. 3328.

31 Günter Spendel, Rechtsbeugung und Justiz, in: JZ 1995, S. 378.

32 BGH, Urt. v. 3.7. 199 I (s StR 209/9I), NJW 1991, S. 2497. Dieses Urteil ist keines gegen einen ehemaligen DDR-Richter, steht aber durchaus mit einem solchen in Zusammenhang: Der hier zur Verhandlung stehende Sachverhalt war die Grundlage fur den zweiten DDR-Rechtsbeugungsprozeß vor dem BGH, in dem das Urteil am 9.5. 1994 erging: BGH, NJW 1994, S. $3238-3242$.

33 Diese Bemerkung bezieht sıch auf die Tatsache, daß die deutlichste und zutreffenste Charakterisierung des NS-Justiz-Systems in der bundesrepublıkanischen Rechtsprechung nicht einem Rechtsbeugungsprozeß entstammt, sondern dem Urtell gegen eine Denunziantın. U. a. war die Rede von der $\leadsto$ Ausnutzung gerichtlicher Formen zur widerrechtlichen Totung*. BGH, Urteil v. 28.6. 1956 (3 StR 366/55), BGHSt 9 , 307.

34 BGH, NJW 1995, S. 3326.

35 Gunter Bemmann, Zu aktuellen Problemen der Rechtsbeugung, in: JZ I 995, S. 1 26. Deshalb plädiert Spendel fur "eine vom Wortlaut ausgehende, auf den objektiven Sinn und Zweck der Gesetzesbestimmung abstellende Auslegung [...]. Der kommunistische Gesetzgeber muß den Anscbein der Rechtsstaatlichkeit, ja Rechtsstaatlichkeıt, den er nach außen zu erwecken suchte, gegen sich gelten lassen, auch wenn sein Rechtssystem sinnerlich،, d. h. nach dem Geist, der es beseelte, verlogen war und anderen Zielen diente. "Spendel, in: JZ 1995, S. 378. Spendel weist auch auf das Urtell des 4. Senats vom 6.10.1994 (BGH, NJW I995, S.66) hin, das zunachst abweichend von den vorangegangenen des 5.Senats fur ene Auslegung der DDR-Gesetze pladıerte, die sıch nicht grundsatzlich von der der Bundesrepublik unterscheiden sollte. Kurze Zert spater spricht der Senat aber von den Wertvorstellungen, in denen die Juristen der DDR verhaftet waren, und billigt ihnen fur das von ihnen gefallte Urteil eine weite Auslegung im Rahmen der Vorgaben der DDR zu. S. a. Gunter Spendel, Rechtsbeugung und BGH - eine Kritzk, in: NJW 1996, S. 81r. 
In den sogenannten Mauerschützenprozessen bedient sich der BGH einer weitaus schlüssigeren und begrüßenswerteren Vorgehensweise als in den Rechtsbeugungsprozessen. Dort überprüfte er die Geltungsvoraussetzungen der entsprechenden DDR-Gesetze an der Verfassung der DDR und den von ihr unterzeichneten völkerrechtlichen Verträgen, ohne darin offenbar einen Verstoß gegen das Rückwirkungsverbot zu sehen, da auch die Anwendung des Grenzgesetzes, nach Ansicht des BGH, Menschenrechtsverletzungen verbot. ${ }^{36}$

Nach dem ersten Mauerschützenurteil sollten diese Maßstäbe sehr wohl auch für die Rechtsprechungsorgane gelten: "Zur Durchführung der sozialistischen Gesetzlichkeit war die Rechtspflege berufen, die die Freiheit, das friedliche Leben, die Rechte und die Würde der Menschen zu schützen hatte. Die Richter sollten nach Art.96 I DDR-Verfassung in ihrer Rechtsprechung unabhängig sein. Hiernach beanspruchten die Gesetze eine Geltung, die nicht durch die Weisungen oder die tatsächliche Staatspraxis bestimmt war. Wer heute den Inhalt der Gesetze der DDR unter der Berücksichtigung der DDR-Verfassung und der Bindung der DDR an internationale Menschenrechtspakte zu ermitteln sucht, unterschiebt demnach nicht dem Recht der DDR Inhalte, die mit dem eigenen Anspruch dieses Rechtes unvereinbar wären. «37

In den Rechtsbeugungsurteilen nimmt sich der BGH wieder zurück. Obwohl der Gerichtshof nicht verkennt, daß das sogenannte politische Strafrecht, einschließlich der Ausreisegesetze und der Pönalisierung öffentlicher Kritik, grundlegenden Verfassungssätzen widersprach, stellt seine Anwendung nach Ansicht des BGH keine "offensichtliche Menschenrechtsverletzung « und keinen Verstoß gegen den »Kernbereich des Rechts « $^{38}$ dar. Erkannt, aber unberücksichtigt bleibt bei den Rechtsbeugungsentscheidungen, daß diese Gesetzgebung rechtsstaatswidrig war und den von der DDR mitunterzeichneten völkerrechtlichen Abkommen, wie dem Internationalen Pakt über bürgerliche und politische Rechte (IPBPR), »im Geist« widersprach. Denn der IPBPR schützt nicht nur mit Art. 6 das Leben, worum es in den Mauerschützenurteilen ging, sondern mit Art. I 2 Abs. 2 auch die Ausreise- und mit Art. 19 die Meinungsfreiheit. Die Begründung, mit welcher der BGH das Menschenrecht auf Leben höher bewertet als andere Menschenrechte, nämlich daß das »menschliche Leben [...] die Basis der Menschenwürde und die Voraussetzung aller anderer Grundrechte « ${ }^{39}$ sei, überzeugt nicht. Der BGH stellt letztlich in der Rechtsbeugungsfrage die Rechtssicherheit der ehemaligen DDR-Juristen über die Unvereinbarkeit der DDR-Gesetze mit den Menschenrechten.

Allerdings hat der BGH inzwischen angedeutet, daß er in Erwägung ziehe, Freiheitsentzug wegen versuchter Republikflucht als Menschenrechtsverletzung aufzufassen. Noch hat er dazu aber keine endguiltige Entscheidung getroffen. ${ }^{40}$ Auch werden bisher Fälle von Pönalisierung öffentlicher Kritik vom BGH nicht proble-

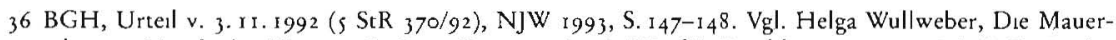
schutzen-Urteile, in: KJ 1993, S. s8-59. Bestatigt durch BVerfG, Beschl.v. 24.10.1996 (2 BvR i $851 / 94$ u. a.).

37 BGH, NJW 1993, S. 146-147. Auf dieser Grundlage hatten auch Rautenberg (Oberstaatsanwalt am BGH) und Burges vor dem ersten Rechtsbeugungsurteıl des Bundesgerichtshofes eine Strafbarkeit fur DDR-Richter angenommen. Erardo Rautenberg/Gerd Burges, Anfangsverdacht wegen Rechtsbeugung gegen Staatsanwalte und Richter der fruberen DDR, in: DiZ 1993, S. 72.

38 BGH, NJW 1995, S. 3327.

39 Ebd.

40 Ebd. S. 3328. Fur die Gleichbebandlung yon Mauerschutzen und Freiheitsberaubung in bezug auf Prozesse wegen versuchter Republikflucht, $\$_{21}$ DDR-StGB: Gerald Grunwald, Die strafrechtlıche Bewertung in der DDR begangener Handlungen, in: StV 1991, S. 35. Gleiche Bewertung von Republikfluchtprozessen und Verfahren gegen Ausreisewvillige: Schaefgen, in: Recht und Polttik 1992, S. 194. 
matisiert, wo die Kritik eigentlich durch das Recht auf freie Meinungsäußerung Art. 19 IPBPR und Art. 27 DDR-Verf. geschützt ist.

Schließlich bleibr es ein Kuriosum der neuen Rechtsprechung zur Rechtsbeugung durch Richter und Staatsanwälte der DDR, daß von einem Grenzsoldaten eine differenziertere und weitblickendere Gesetzesauslegung erwartet wird als von ausgebildeten Juristen. Der Grenzsoldat soll eine menschenrechtsfreundlichere Auslegung der DDR-Gesetze anhand höherrangigen DDR-Rechts yornehmen, obwohl er unter dem Zwang einer schnellen Entscheidung stand und ungeklärt ist, ob er sich dieser Möglichkeit überhaupt bewußt war. Der BGH sieht darin noch nicht einmal einen Schuldminderungsgrund. Dem ausgebildeten Juristen möchte der BGH Vergleichbares noch nicht einmal zumuten, wenn Zeit zur Überlegung reichlich vorhanden war.

\section{Die Umsetzung der Maßstäbe in den Entscheidungen des $B G H$}

Den inzwischen über einem Dutzend BGH-Entscheidungen zu Rechtsbeugungen in der DDR lagen strafrechtliche und zivilrechtliche Ausgangsfälle zugrunde. Im folgenden soll es vor allem um erstere gehen.

1995 bestätigte der Fünfte Senat die Verurteilung eines ehemaligen Richters des Obersten Gerichts der DDR wegen Rechtsbeugung in Tateinheit mit Totschlag und versuchtem Totschlag zu einer Gesamtfreiheitsstrafe von drei Jahren und neun Monaten mit einer Begründung, die an Klarheit und Schärfe nichts vermissen läßt: »Hier wird das angesichts des festgestellten tatsächlichen Sachverhalts fehlende Gewicht des von einem Angekl. verschuldeten Unrechts durch übersteigerte, nur noch propagandistisch zu verstehende Formulierungen überspielt. In einem so begründeten Todesurteil kommen schon in der Wortwahl unmißverständlich der unbedingte Wille zur physischen Vernichtung eines politischen Gegners ohne Rücksicht auf dessen persönliche Schuld [...] auch um den Preis eines Menschenlebens zum Ausdruck. Dies ist willkürliches Töten unter dem Vorwand eines justizförmigen Verfahrens. «"

Andererseits handelt es sich bei der Mehrzahl der Verfahren, die sich mit Rechtsbeugung in der ehemaligen DDR befassen, nicht um die oben angesprochenen krassesten Auswüchse der DDR-Justiz. Der Großteil der DDR-Urteile, in denen Bürger zu Opfern dieser Rechtsprechung wurden, wird von Verfahren gebildet, in denen "nur « die Freiheit entzogen wurde. Mit welchen Repressalien dieser Freiheitsentzug verbunden war, wird von Schroeder anschaulich geschildert: Überfüllung der Gefängnisse, katastrophale hygienische Zustände, harte Arbeitseinsätze und scharfe Zwangsmaßnahmen. ${ }^{42}$

Vor diesem Hintergrund sind die Bagatellverfahren zu sehen, in denen Strafen verhängt wurden, denen jeglicher Bezug zur »Tat« fehlte, die aber trotzdem in der Regel vom BGH nicht als Rechtsbeugung verurteilt wurden. Besonders kritisch zu sehen ist die Entscheidungspraxis des BGH im Bereich des politischen Strafrechts. Denn gerade dieses war das spezifische Instrument der DDR-Machteliten zur Unterdrükkung und Drangsalierung politisch Andersdenkender oder gänzlich unpolitischer Ausreisewilliger, die nicht nur den von der DDR mitunterzeichneten Menschenrechtsverträgen, sondern auch der Verfassung der DDR widersprach.

1994 befaßte sich der BGH mit dem Fall eines Richters und einer Staatsanwältin, die Mitte der achtziger Jahre einen Mann zu einer Freiheitsstrafe von einem Jahr und

41 BGH, NJW 1996, S. 861.

$42 \mathrm{Vgl}$. Schroeder, in: DR1Z 1996, S. 81. 
sechs Monaten verurteilt hatten. Da er auf legalem Wege nicht ausreisen durfte, hatte der Mann seinen Prozeß provoziert, indem er bei einer öffentlichen Veranstaltung ein Schild mit der Aufschrift: "DDR! Deine Grenzen sind für mich kein Friedensbeitrag!« über seinen Kopf gehalten hatte. Die Strafe für diese »Beeinträchtigung staatlicher oder gesellschaftlicher Tätigkeit « nach $\$ 214$ DDR-StGB war, auch vom BGH anerkannt, hoch angesetzt. Aber letzten Endes wurde er, wie erhofft, in die Bundesrepublik abgeschoben und seine Familie freigekauft. Der BGH sieht in dem Verfahren noch keine Rechtsbeugung, weil die Einbindung der Angeklagten in das System der DDR unter Hinweis auf die scharfen Vorschriften gegen Ausreisewillige noch nicht strafbar sei. Strafbarkeit "setze vielmehr erst dann ein, wenn Strafvorschriften ausschließlich als Vorwand für Zwangsmaßnahmen zur Unterdrückung des - auch nach dem Recht der DDR nicht strafbaren - Begehrens nach Ausreise benutzt wurden. Dies ist - wie vorstehend ausgeführt - angesichts der bewußt provokanten Handlungsweise des P, der Opfer des Systemunrechts geworden ist, hier nicht ersichtlich.«43

Die zweite Entscheidung zum politischen Strafrecht vom i 5.9. 1995 gilt gleichzeitig als Grundsatzurteil. ${ }^{44}$ Hier wurde gegen eine Staatsanwältin verhandelt, die für Straftaten im Bereich des politischen Strafrechts und sogenannte Staatsverbrechen zuständig war. Sie war vom LG Berlin als Anklagevertreterin ${ }^{45}$ in zehn Fällen wegen Rechtsbeugung in Tateinheit mit Freiheitsberaubung zu drei Jahren Haft verurteilt worden. Die Angeklagte hatte mit ihrer Revision vor dem BGH größtenteils Erfolg.

So war nach Ansicht des BGH die Verurteilung eines Chemikers wegen »staatsfeindlicher Hetze« $(\$ 106$, teilweise i. V.m. $\ 108$ DDR-StGB) zu zwei Jahren Gefängnis rechtmäßig. Dieser hatte während des Unterrichts vor Oberschülern »die DDR, ihre Repräsentanten und mit ihr verbündete Staaten herabgewürdigt «. ${ }^{6}$ Meinungsäußerungen seien in der DDR nur geschützt gewesen, wenn es sich um von der SED gewünschte gehandelt habe. Die Strafe sei zwar unverhältnismäßig hoch gewesen, als grob ungerecht und schwerer Verstoß gegen die Menschenrechte im Sinne willkürlicher Rechtsanwendung erscheine sie jedoch im Blick auf die Tatumstände nicht. ${ }^{47}$

Die Verurteilung einer Frau wegen vorbereitetem und versuchtem »ungesetzlichen Grenzübertritts « $\left(\mathbb{2}\right.$ I $_{3}$ DDR-StGB $)$ zu zwei Jahren und sechs Monaten Gefängnis war aus der Sicht des BGH ebenfalls rechtmäßig. Es bestand nach Ansicht des BGH kein Rechtsanspruch auf eine Ausreise und in der Anwendung von $\mathbb{2}$ I $_{3}$ DDRStGB keine Überdehnung der Strafvorschrift. Das LG habe »in unzulässiger Weise die Wertvorstellungen einer rechtsstaatlichen Verfassung ${ }^{48}{ }^{8}$ zugrundegelegt. Der Senat habe zwar noch nicht entschieden, ob der Freiheitsentzug bei versuchter Republikflucht als Menschenrechtsverletzung zu werten sei ${ }^{49}$, in dem vorliegenden Fall sei

43 BGH, NJW 1995, S. 67

44 BGH, NJW I995, S. 3324-3332. Die im folgenden exemplarsch geschilderten Sachverhalte sind typische Beıspiele fur den Justizalltag in der DDR ım Bereıch des polıtıschen Strafrechts. An diesem Tag fallte der BGH vier weitere Urteile, von denen zwe nicht zur Veroffentlichung vorgesehen worden sind, s StR 642/94 und S StR 68/95, wahrend zwel andere in stark gekurzter Version in der NJ 1996, S. 1 52-153 erschienen sind. Es handelt sich um weitere Anklagen von Rıchtern und Staatsanwalten wegen "Ponal ${ }_{1-}$ sıcrung offentlicher Kritik“ und justızıeller Diskrıminierung Ausreısewilliger. Ebenfalls in diesem Rahmen bewegen sich die Urtelle des 4. Senats vom 30.11. 1995, NStZ-RR 1996, S.65-71.

45 Zur Stellung von Staatsanwalten gegenuber Richtern in der ehemaligen DDR: Wolfgang Behlert, Organisation und sozıaler Status der Rıchter und Rechtsanwalte in der DDR, in: K] 1991, S. 184-197. ${ }_{46}$ BGH, NJW 1995, S. 3328.

47 Ebd.

48 Ebd.

49 Vgl. a. BGH, Beschluß v 8.2. 1995 (s StR ${ }_{187 / 95}$ ), NStZ 1995, S. 289. 
die Strafe jedenfalls durch den mehrfachen Fluchtversuch noch nicht rechtsbeugerisch überhöht.

Weiter akzeptierte der BGH die Verurteilung eines Ehepaares, das zu zwei Jahren bzw. zwei Jahren und drei Monaten Freiheitsstrafe verurteilt wurde, weil sie Material über ihre Ausreiseanträge verschickt bzw. in ihren Ausreiseanträgen mit einer Veröffentlichung gedroht hatten. Die Auslegung als »staatsfeindliche Verbindung"

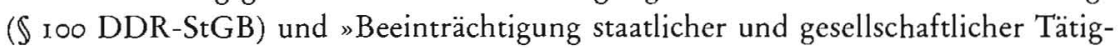
keit $(\$ 214$ DDR-StGB) sei eine letztlich "hinnehmbare Interpretation der Vorschriften ${ }^{{ }^{\circ}}$ und damit keine Rechtsbeugung. Das krasse Mißverhältnis von Tat und Sanktion bilde keinen unerträglichen Willkürakt.

Die Verurteilung zweier Brüder aus der Bundesrepublik zu drei Jahren und sechs Monaten bzw. einem Jahr und sechs Monaten wurde vom BGH ebenfalls unbeanstandet gelassen. Sie hatten einem DDR-Bürger ihre Papiere gegeben und den Weg durch die Grenzanlage erklärt. Dieser hatte daraufhin die DDR verlassen. Trotz der Bedenken aus "systematischen und teleologischen Gründen «, die beiden wegen "Menschenhandels« nach $\mathbb{1}$ 132 I DDR-StGB anzuklagen, und der äußerst hohen Strafen sei angesichts der geglückten Flucht des DDR-Bürgers keine Rechtsbeugung anzunehmen. ${ }^{\text {st }}$

Auch die Verurteilung eines jungen DDR-Bürgers zu einem Jahr Gefängnis, weil er in einem öffentlichen Gebäude erklärt hatte, falls sein Ausreiseantrag abgelehnt werde, würde er sich mit dem Schild »Ich will endlich ausreisen « vor das Staatsratsgebäude oder an die Staatsgrenze stellen, blieb vor dem BGH straffrei. Die Qualifizierung dieses Verhaltens als »Beeinträchtigung staatlicher Tätigkeit« ( $\$ 2 I_{4} \mathrm{Abs}$. I DDR-StGB) liege noch innerhalb der Normgrenzen, weil die Äußerung »als Drohung - nämlich als Eingrenzung der Entscheidungsmöglichkeit staatlicher Organe $\aleph^{\zeta 2}$ - ausgelegt werden könne. Auch das krasse Mißverhältnis von Schuld und Sanktion bilde noch keine Rechtsbeugung.

Im Sinne der zuyor entwickelten Beurteilungsmaßstäbe mag dieser Schluß rein formaljuristisch nachvollziehbar sein. Aber die Anerkennung eines Systemunrechts als rechtmäßige Entscheidung durch den BGH zeigt auch die Zwickmühle, in die eine solche Argumentation führt. Schroeder spricht von einer »karikaturreifen Rolle «'3, in die sich das Gericht dadurch begibt, daß es die DDR-Normen in peinlich genauer Art und Weise berücksichtigt und zur Anwendung bringt. Besser habe es ein DDRGericht auch nicht machen können. ${ }^{54}$

Schließlich blieb vor dem BGH auch die Verurteilung eines Mannes unbeanstandet, der in einem Fußgängertunnel ein Schild zur Schau stellte, auf dem stand: „Seit I2 Monaten werde ich am legalen Verlassen der DDR gehindert«, und dafür eine Freiheitsstrafe von einem Jahr und drei Monaten erhielt. Die Auslegung des $\$ 214$ Abs. I DDR-StGB als "Mißachtung der Gesetze« hielt sich nach Ansicht des BGH noch »in den Grenzen möglicher Auslegung “'s. Die verhängte Strafe grenze zwar an einen Willkürakt, weil der Tatbestand nur bei extensiver Auslegung der Norm erfüllt sei, aber trotz dieser zwei schwerwiegenden Feststellungen sei der Tatbestand der Rechtsbeugung noch nicht erfüllt. "Immerhin hatte sich der Betr. nach wiederholter Ablehnung von Ausreiseanträgen und nach ausdrücklicher Vorwarnung, folglich in

so BGH, NJW 1994, S. 3329 .

51 Ebd., S. 3329-3330.

52 Ebd., S. 3330.

53 Schroeder, in: DRiZ 1996, S. 87.

s4 Ebenso Spendel, in: NJW 1996, S.811.

is BGH, NJW 1995, S. 3329. 
voller Kenntnis ihm drohender massiver strafrechtlicher Konsequenzen, zur Durchführung seiner Aktion entschlossen. ${ }^{56}$

Die Schieflage dieser Argumentation scheint dem Senat bewußt gewesen zu sein. Denn er gesteht in diesem Zusammenhang ein, wie unbefriedigend ein solches Urteil ist, um dann aber den verhängnisvollen Nachsatz zu bilden, daß die DDR-Justiz aus ihrer Sicht das Verhalten von Menschen mit Zivilcourage - wie die Ereignisse vom November 1989 zeigten $-z u$ Recht fürchten mußte.

Muß der BGH in einem Rechtsbeugungsprozeß tatsächlich das Recht der DDRJustiz und damit des Staates »DDR « auf seine »Selbstbehauptung « schützen, wenn der verurteilte DDR-Bürger nach rechtsstaatlichen Maßstäben als völlig unschuldig anzusehen wäre? Muß der Schutz der innerstaatlichen Rechtspflege ${ }^{57}$ auch im Fall der DDR höher bewertet werden als der Schutz individueller Freiheitsrechte bei DDR-Bürgern, wenn es um Rechtsbeugung geht? ${ }^{58}$

In drei anderen Fällen ${ }^{59}$ erkannte der BGH im selben Urteil allerdings auf Rechtsbeugung. Konstruiert und unverständlich bleibt hierbei jedoch die Abgrenzung zu den übrigen Fällen: So war eine Frau zu einer Haftstrafe von einem Jahr verurteilt worden, weil sie sich vor dem Mikrophon eines ARD-Korrespondenten zu einer kritischen Bemerkung hatte hinreißen lassen. Der BGH billigt ihr ein Spontanverhalten zu, so daß nach DDR-Recht Strafen unterhalb der Freiheitsstrafe angemessen gewesen wären.

In einem zweiten Fall befand sich eine Sechzehnjährige wegen »öffentlicher Herabwürdigung « $(\$ 220$ DDR-StGB) in Untersuchungshaft, weil sie eine geringe Zahl von Aufrufen verteilt hatte, die sich auf die Erhaltung der Punk-Szene in Ost-Berlin bezogen. Der BGH sprach dem Vergehen Bagatellcharakter zu. Da mildernde Umstände der Subsumtion unter den angewandten Paragraphen nicht vorgesehen gewesen seien, liege wegen falscher Zuordnung eine Rechtsbeugung vor.

Im dritten Fall war ein Mann zu einem Jahr und zwei Monaten verurteilt worden, weil er nach der Ablehnung seines Ausreiseantrags unter Vorlage eines DDR-Passes an der Grenze seine Ausreise verlangt hatte. Die Anklage wegen der »Beeinträchtigung staatlicher Tätigkeit « $(\mathbb{2} 214$ Abs. I DDR-StGB) hielt der BGH für unzulässig, weil sich zur Zeit der Tat keine weiteren Personen an der Grenze aufgehalten und keine Anzeichen von Gewalt oder Drohung vorgelegen hätten. Auch stehe die Strafe in einem unerträglichen Mißverhältnis zur "Tat«.

In dem Urteil des 3. Senats vom I s. I I . I995 hielt der BGH der Vorinstanz, dem LG Dresden, sogar vor ${ }^{60}$, dem Rechtsbeugungstatbestand "zu enge Grenzen « gesetzt zu haben. Hatte das LG Dresden den Angeklagten freigesprochen, so erkannte der Senat in acht von zehn Fällen auf Rechtsbeugung. So erfreulich diese Feststellungen auf der einen Seite sind, eine Korrektur der zuvor kritisierten Entscheidungen enthalten sie nicht.

$56 \mathrm{Ebd}$.

57 BGH, NJW 1995, S. 3324 .

58 S. zum Rechtsgut des Rechtsbeugungsparagraphen ausfuhrlich die Dissertation von Frank Scholderer, Rechtsbeugung ım demokratischen Rechtsstaat. Zur Rekonstruktıon des $\$ 3{ }_{3} 6 \mathrm{StGB}$ für die Gegenwart, Baden-Baden 1993, S. 503-504.

59 BGH, NJW I995, S. 3330-3332.

60 BGH, DtZ i 996, S. 94. 
In den meisten Fällen werden die Opfer der DDR-Justiz rehabilitiert worden sein, denn es sind gerade diese Rehabilitationsverfahren, die von Amts wegen eine Untersuchung auf Rechtsbeugung einleiten. ${ }^{61}$ Den zugrundeliegenden Verurteilungen jedoch wird durch den höchstrichterlichen Entscheid eines Rechtsstaates weiterhin attestiert, sie hätten sich im Rahmen des rechtlich Gestatteten gehalten.

Dem BGH fällt es offenbar schwer, Maßstäbe zu finden und anzuwenden, die dem repressiven Mißbrauch von Gesetzen in der DDR gerecht werden. Ursächlich ist m. E. vor allem der Ausgangspunkt der BGH-Rechtsprechung, daß das politische Strafrecht der DDR den »Kernbereich des Rechts « nicht verletze. Durch diese Argumentation bleiben die entsprechenden Paragraphen wirksam, und der BGH verbaut sich selbst den Rückgriff auf höherrangiges Recht, nämlich die Anwendung der DDR-Verfassung und der völkerrechtlichen Verträge, die die DDR unterzeichnet hat. Deshalb kann der BGH den repressiven Charakter der politischen Rechtsprechung zwar erkennen, jedoch nur, um ihm anschließend über seine Maßstabsbildung doch zu exkulpieren.

So läßt die Praxis von dem Versprechen, mit über 12000 Ermittlungsverfahren dem Unrecht in der ehemaligen DDR Herr werden zu wollen, wenig mehr als den Schein. Die Rechtsprechung des BGH zu den Rechtsbeugungsverfahren hat bisher eine wahre Einstellungsflut ${ }^{62}$ zur Folge gehabt, die zuweilen groteske Züge annimmt. So zitiert Spendel einen Fall vor dem LG Berlin, in dem das Gericht entgegen seiner Überzeugung »von der >schweren Schuld der angeklagten SED-Funktionäre von deren Verurteilung abgesehen hat, weil diese vom BGH auf Grund seiner abzulehnenden Judikatur ja doch aufgehoben würde $\ll_{.}{ }_{3}$

In der Sache handelt es sich damit um eine Art Amnestie für die Masse der DDRRichter und Staatsanwälte. Für geringfügigere Straftaten in der DDR wäre eine Amnestie gewiß zu erwägen, aber nicht speziell und allein für Justizdelikte. Eine Amnestie hätte auch der Rechtsunsicherheit Rechnung tragen können, die sich daraus ergibt, daß sich die rechtlichen Maßstäbe der Bundesrepublik nicht bruchlos auf die DDR-Vergangenheit anwenden lassen. So ist es zu einer Überforderung der BGH-Rechtsprechung gekommen, die sich nun in eine Art Teillegitimation von DDR-Verhältnissen verstrickt hat.

\section{Joachim Perels Späte Entlegitimierung der NS-Justiz}

Sieben Jahre existierte die Bundesrepublik, als ihr oberstes Strafgericht den Vorsitzenden SS-Richter eines Standgerichts im Konzentrationslager Flossenbürg, Otto Thorbeck, der Dietrich Bonhoeffer, Hans Oster, Admiral Canaris und andere Widerstandskämpfer wegen angeblichen Landesverrats und mindestens teilweisen Hochverrats am 8. April I945 zum Tode verurteilt hatte, rechtskräftig freisprach.

6I Rautenberg/Burges, in: DtZ r993, S. 71 .

62 Die aktuelle Zahl der Enstellungen liegt nicht vor, aber im August 1994 hatte erst ein Verfahren rechtskraftig mit einer Verurteilung geendec und stand $1 \varsigma_{23}$ Einstellungen gegenuber (BT-Dr. $12 / 8402$, S. 4.) S. a. u. a. das Interview mit der Staatssekretärin der Justiz Sachsen-Anhalts, in: Die Zeit v. 23.6. 1995, S. 12 .

63 Spendel, in: NJW 1996, S.810. 